\title{
Activation of STAT5 contributes to proliferation in U87 human glioblastoma multiforme cells
}

\author{
CUI FENG $^{1}$ and SHOUQIANG CAO ${ }^{2}$ \\ Departments of ${ }^{1}$ Anesthesiology and ${ }^{2}$ Neurosurgery, Fourth Affiliated Hospital of Harbin Medical University, \\ Harbin, Heilongjiang 150001, P.R. China
}

Received July 5, 2013; Accepted March 25, 2014

DOI: $10.3892 / \mathrm{mmr} .2014 .2223$

\begin{abstract}
Rapid increases in the tyrosine phosphorylation of signal transducers and activators of transcription 5 (STAT5) proteins have been extensively documented in cells stimulated with cytokines and growth factors. However, the mechanisms by which STAT5 translocates to the nucleus and regulates proliferation in human glioblastoma multiforme cells have not been studied in detail. To the best of our knowledge, the present study demonstrated for first time that stimulation of a glioblastoma multiforme (GBM) cell line (U87-MG) with hepatocyte growth factor (HGF) resulted in the phosphorylation of STAT5 at Tyr-694/699 and nuclear translocation of STAT5. In addition, HGF promoted nuclear translocation of STAT5 in a time-dependent manner and increased the proliferation of U87-MG cells. In order to determine the role of STAT5 directly, RNA interference was used to knockdown STAT5 expression in the U87-MG cell line. It was illustrated that small interfering RNA (siRNA) against STAT5 successfully inhibited the protein expression of STAT5 in the U87-MG cell line, leading to a potent suppression of tumor cell proliferation with or without HGF treatment. In order to broaden the investigation and to determine the role of STAT5 in vivo, immunohistochemistry (IHC) was applied to evaluate STAT5 expression in 100 newly diagnosed glioma and 10 non-neoplastic brain tissues. p-STAT5 expression increased according to the histopathological grade of the glioma. However, no p-STAT5 staining was observed in non-neoplastic brain tissues. These findings suggested that inhibition of the STAT5 pathway may be an effective therapeutic strategy for the clinical management of GBM.
\end{abstract}

Correspondence to: Dr Cui Feng, Department of Anesthesiology, Fourth Affiliated Hospital of Harbin Medical University, 37 Yi Yuan Street, Harbin, Heilongjiang 150001, P.R. China

E-mail: mdcuifeng@gmail.com

Key words: glioblastoma multiforme, STAT5, p-STAT5, small interfering RNA, tumor proliferation

\section{Introduction}

Signal transducers and activators of transcription 5 (STAT5) can be activated by numerous cytokines and growth factors in various cell lines and tissues. Furthermore, STAT5 has a variety of regulatory roles, which control different cell functions, including growth, survival, differentiation and invasion (1). However, the role of STAT5 in the pathogenesis of glioblastoma multiforme (GBM) has not been examined fully and the function of hepatocyte growth factor (HGF), the physiological activator of STAT5, in stimulating STAT5 in GBM cells remains to be elucidated. The present study, to the best of our knowledge, demonstrated for the first time that HGF induces phosphorylation of STAT5 at Tyr-694/699, nuclear translocation of STAT5 and increases proliferation of the U87-MG cell line. To directly assess the biological significance of STAT5 signaling in GBM cells, using small interfering RNA (siRNA) to deplete STAT5 in the human GBM cell line (U87-MG) and to inhibit the HGF-induced STAT5 activation, changes in cell viability and cell cycle progression were examined. Changes in the expression of several genes, including Cyclin D1, p21 and p27, that are directly associated with cell cycle regulation were also assessed. The aim of the present study was to determine the role of STAT5 signaling in GBM progression and to test the hypothesis that STAT5 signaling may serve as a therapeutic target.

\section{Materials and methods}

Cell culture. The human U87-MG cell line was obtained from the Cell Bank of Type Culture Collection of the Chinese Academy of Sciences (CBTCCCAS; Shanghai, China). The human U87-MG cell line was cultured in Dulbecco's modified Eagle's medium (DMEM; Gibco-BRL, Carlsbad, CA, USA) supplemented with $2.0 \mathrm{~g} / 1$ sodium bicarbonate and $10 \%$ fetal bovine serum (FBS; Gibco-BRL) in a humidified atmosphere containing $5 \% \mathrm{CO}_{2}$ and $95 \%$ air at $37^{\circ} \mathrm{C}$. For experimental purposes, confluent cultures of U87-MG cells were serum-starved for $12 \mathrm{~h}$ prior to treatment with $40 \mathrm{ng} / \mathrm{ml}$ HGF (R\&D Systems, Minneapolis, MN, USA).

Cell stimulation with HGF. Confluent cultures of U87-MG cells were washed twice with DMEM without 
serum, equilibrated in the same medium at $37^{\circ} \mathrm{C}$ for at least $30 \mathrm{~min}$ and then collected at different time-points $(0,15,30$, $60,120,180$ and $240 \mathrm{~min}$ ) following HGF treatment. A total of $2 \times 10^{6}$ cells was grown in $60 \mathrm{~mm}$ dishes containing $4 \mathrm{ml}$ of DMEM for each experimental condition.

Transient transfection of STAT5 siRNA. siRNA oligos for knockdown of endogenous STAT5 proteins were prepared by using the ON-TARGETplus SMARTpool siRNA from Dharmacon, Inc. (Lafayette, CO, USA). Cells were transfected with STAT5 siRNA (100 $\mathrm{nM})$ by using the DharmaFECT siRNA transfection reagent (Dharmacon, Inc.) according to the manufacturer's instructions. ON-TARGETplus non-targeting siRNA (Dharmacon, Inc.) was used as a negative control (control siRNA) and the selective silencing of STAT5 was confirmed by western blot analysis.

Cellular protein preparation and western blot analysis. U87-MG cells were treated as described. Nuclear cell protein for studying p-STAT5 was extracted with the ProteoJET ${ }^{\mathrm{TM}}$ Cytoplasmic and Nuclear Protein Extraction kit (Fermentas, Vilnius, Lithuania) according to the manufacturer's instructions. Total cell protein was extracted with the Total Cell Protein Extraction kit (Millipore, Billerica, MA, USA). Protein concentrations were determined using the Coomassie (Bradford) protein assay kit (Thermo Fisher Scientific, Waltham, MA, USA). Equal amounts of protein were separated by SDS-PAGE using $8 \%$ separating gels followed by transfer to nitrocellulose membranes. Following transfer, membranes were blocked using 5\% non-fat dried milk in phosphate-buffered saline (PBS; pH 7.2) and incubated overnight at $4^{\circ} \mathrm{C}$ with the primary antibody (pAb), including STAT5, p-STAT5a/b, Cyclin D1, p21, p27 and $\beta$-actin (1:1,000 dilution; Santa Cruz Biotechnology, Inc., Dallas, TX, USA). The membranes were washed three times with PBS, $0.1 \%$ Tween 20 and then incubated with secondary Abs (horseradish peroxidase-conjugated, goat antibodies to rabbit and goat antibodies to mouse; Santa Cruz Biotechnology, Inc., Santa Cruz, CA, USA; dilution of 1:5,000) for $2 \mathrm{~h}$ at $24^{\circ} \mathrm{C}$. Following washing three times with PBS/0.1\% Tween 20, the immunoreactive bands were visualized using enhanced chemiluminescence detection reagents. Autoradiograms were scanned and the labeled bands were quantified using the Sigma-Gel software (Sigma, St. Louis, MO, USA).

RNA isolation and quantitative polymerase chain reaction $(q P C R)$. Total RNAs from cells were extracted and cDNA synthesis and amplification were performed as described previously (2). Primers were designed as: p $21^{\text {Cip1 }}$ forward, 5'-CGATGCCAACCTCCTCAACGA-3' and reverse, 5'-TCGCAGACCTCCAGCATCCA-3'; p27 ${ }^{\mathrm{Kip} 1}$ forward, 5'-TGCAACCGACGAT TCTTCTACTCAA-3' and reverse, 5'-CAAGCAGTGATGTATCTGATAAACAAGGA-3'; CyclinD1 forward, 5'-AACTACCTGGACCGCTTCCT-3' and reverse, 5'-CCACTTGAGCTTGTTCACCA-3'; GAPDH forward, 5'-GACTCATG ACCACAGTCCATGC-3' and reverse, 3'-AGAGGCAGGGATGATGTTCTG-5'. Comparative qPCR was performed in triplicate, including no template controls. Relative expression was calculated using the comparative $\mathrm{Ct}$ method.
MTT assay for cell viability. U87-MG cells (5x10 3 per well) were incubated in 96-well plates each containing $200 \mu \mathrm{l}$ of medium and cultured overnight in growth medium. Then the culture medium was replaced with DMEM supplemented with $10 \% \mathrm{FBS}$ and HGF $(40 \mathrm{ng} / \mathrm{ml})$. To assay the effect of STAT5 knockdown on cell proliferation, cells were transfected in 96-well plates. The rate of cellular proliferation was measured every $24 \mathrm{~h}$ for $96 \mathrm{~h}$. At the end of each time-point, $20 \mu \mathrm{l}$ of $5 \mathrm{mg} / \mathrm{ml}$ of MTT (Sigma) was added to each well and incubated for $4 \mathrm{~h}$ at $37^{\circ} \mathrm{C}$. Following removal of the culture medium from each well, $150 \mu \mathrm{l}$ of dimethylsulfoxide was added to the MTT-treated wells and the absorption at $570 \mathrm{~nm}$ was determined using an ELISA spectrophotometer (model 3550; Bio-Rad Laboratories, Richmond, CA, USA). Each experimental condition was conducted in triplicate.

Cell cycle analysis. Approximately $1 \times 10^{6}$ cells were harvested at specified time-points, washed twice with PBS and fixed in cold ethanol for $12 \mathrm{~h}$ at $4^{\circ} \mathrm{C}$ and then incubated with propidium iodide for $30 \mathrm{~min}$. Cells were treated with siRNA as described. Following $72 \mathrm{~h}$, the cells were added to a conical tube and spun at $1,000 \times \mathrm{g}$ for $3 \mathrm{~min}$. The pellet was vortexed at a low speed and $0.5 \mathrm{ml}$ of cold PBS was added. Then, the pellet was vortexed again for 2-3 sec and resuspended in $5 \mathrm{ml}$ of cold PBS. The cells were centrifuged for $6 \mathrm{~min}$ at 1,000 $\mathrm{x} \mathrm{g}$ and the PBS was then aspirated off. Cold PBS $(0.5 \mathrm{ml})$ was added and pipetted up and down to achieve a single cell suspension. The tube was prepared with $4.5 \mathrm{ml}$ ice-cold $100 \%$ ethanol. Cells $(0.5 \mathrm{ml})$ were permeablized by adding ice-cold $100 \%$ methanol slowly to pre-chilled cells whilst gently vortexing. Subsequently, cells were incubated on ice or at $4^{\circ} \mathrm{C}$ for $12 \mathrm{~h}$ and then stained with propidium iodide. Thereafter, cells were analyzed using a flow cytometer (BD FACSCanto II; BD Biosciences Franklin Lakes, NJ, USA).

Reporter gene assay. The pGL3-CyclinD1 vector and the control vector were prepared as described previously (3). Briefly, $0.4 \mu \mathrm{g}$ of reporter gene constructs was transfected into U87-MG cells using Lipofectamine (Invitrogen Life Technologies, Carlsbad, CA, USA) reagent according to the manufacturer's instructions. This transfection was performed concurrently with the transfection of STAT5 siRNA. Cells co-transfected with pRL-TK served as controls. The results are expressed as the percentage of relative luciferase activity of the control group without HGF stimulation, which was set to 1 .

Immunofluorescence microscopy. U87-MG cells were grown on coverslips, washed with serum free-DMEM and treated with $40 \mathrm{ng} / \mathrm{ml} \mathrm{HGF}$ for $1 \mathrm{~h}$ at $37^{\circ} \mathrm{C}$. The cells were fixed for $10 \mathrm{~min}$ with $4 \%$ paraformaldehyde in PBS, permeabilized with $0.5 \%$ Triton X-100 in PBS and blocked for $1 \mathrm{~h}$ in $5 \%$ fetal bovine serum in PBS. Cells were incubated overnight at $4^{\circ} \mathrm{C}$ with p-STAT5 Ab (1:100 dilution), as indicated, followed by fluorescein isothiocyanate (FITC)-labelled anti-rabbit secondary $\mathrm{Ab}$ for 2 h. Finally, Hoechst (1:1,000 dilution) was used for nonspecific staining of the nucleus. Cells were viewed using a confocal microscope (Leica, Mannheim, Germany).

Tissue samples and patients. Tumor specimens were obtained from patients admitted for diagnosis and treatment at the Fourth 
Affiliated Hospital of Harbin Medical University (Harbin, China). The diagnosis was made according to World Health Organization criteria. The present study was approved by the ethics committee of the Fourth Affiliated Hospital of Harbin Medical University and was based on the criteria of the Helsinki convention. Approval for use of the tissue in the present study was obtained from the institutional review board. Fresh surgical samples from glioma patients and non-neoplastic brain tissues (temporal lobectomy from epilepsy surgery) were immediately snap-frozen in liquid nitrogen upon surgical removal. The formalin-fixed, paraffin-embedded archival tissue blocks were retrieved and the matching HE-stained slides were screened for representative tumor regions by a neuropathologist. The tissue included 25 diffuse astrocytomas (grade II), 25 anaplastic astrocytomas (grade III) and 50 glioblastomas (grade IV). In addition, ten non-neoplastic brain tissues from epilepsy surgical resections were also included.

Immunohistochemical (IHC) analysis. The perfused brains were cryoprotected in a solution of $20 \%$ sucrose in $0.1 \mathrm{M}$ of potassium phosphate buffer overnight. The brain sections were cut on a freezing microtome (Leica SM2000 R) and mounted on gelatinized slides. The sections were dried at $40-50^{\circ} \mathrm{C}$ for $2 \mathrm{~h}$ and were maintained at $-20^{\circ} \mathrm{C}$ until analysis. IHC analysis followed as described briefly: The sections were incubated at room temperature overnight with the primary antibody (p-STAT5, 1:100) diluted in PBS with Tween 20 (PBST). The negative controls received only PBST. The slides were washed with PBST and incubated with the secondary antibodies (1:1,000 in PBST) for $90 \mathrm{~min}$. The slides were washed again with PBST and incubated with streptavidin-horseradish peroxidase (1:200 in PBST) for $60 \mathrm{~min}$. The reactions were developed with $0.04 \%$ 3,3'-diaminobenzidine (DAB) $+0.03 \%$ $\mathrm{H}_{2} \mathrm{O}_{2}$. The DAB reactions were intensified with an $\mathrm{OsO}_{4}$ solution $(0.04 \%)$ for $30 \mathrm{~min}$. The slides were counterstained with hematoxylin, dehydrated and mounted with Permount. Results were visualized and images were captured under a light microscope (Olympus BX-51; Olympus Optical, Tokyo, Japan).

The degree of immunostaining of sections was viewed and scored separately by two independent investigators, the scores were determined by combining the proportion of positively stained tumor cells and the intensity of staining. Scores from the two investigators were averaged for further comparative evaluation of the p-STAT5 expression. The proportion of positively stained tumor cells was graded as described previously (4).

Statistical analysis. All the data are presented as the mean \pm standard deviation. All analyses were performed with one-way analysis of variance using SPSS 13.0 software (SPSS, Inc., Chicago, IL, USA). P<0.05 was considered to indicate a statistically significant difference.

\section{Results}

HGF induces nuclear translocalization of STAT5. Activated STATs are known to translocate from the cytoplasm to the nucleus (5). Immunofluorescent analysis of treated and untreated U87-MG cells indicated that p-STAT5 accumulated in the nucleus with HGF treatment (Fig. 1). HGF preferentially
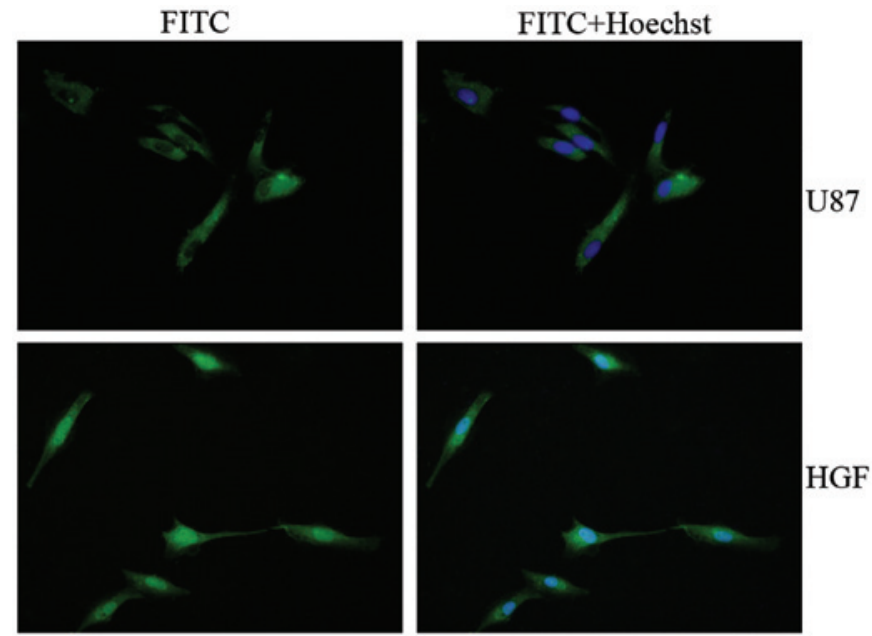

Figure 1. Immunofluorescence staining of U87-MG glioma cells with and without HGF treatment. Upper panels, no HGF stimulation; lower panels, HGF stimulation. Left panels, p-STAT5 staining, right panels, p-STAT5 and nucleus staining. p-STAT5, phosphorylated signal transducers and activators of transcription 5; HGF, hepatocyte growth factor.

activated STAT5, which subsequently translocated to the nucleus. This observation prompted us to examine whether STAT5 was constitutively activated in glioma tissues.

STAT5 is constitutively activated in GBM but inactivated in the U87-MG cell line. To determine whether STAT5 is constitutively activated in glioma, the levels of p-STAT5 expression were assessed by immunohistochemistry using anti-p-STAT5a/b (Tyr694/Tyr699) antibody on glioma tissues. Among glioma samples $92 \%$ demonstrated positive nuclear staining, whereas normal tissue did not stain. The p-STAT5 staining was predominantly nuclear in vivo (Fig. 2). There was no significant difference in constitutive activation frequency between low and high grade gliomas. However, the expression levels of p-STAT5 were significantly higher in high grade gliomas (grade III and grade IV) compared with low grade gliomas (grade II) $(\mathrm{P}<0.05)$, which supports the hypothesis that STAT5 activation is associated with the progression of glioma.

Western blot analysis with anti-STAT5 and anti-p-STAT5 antibodies revealed that STAT5 was inactivated in the U87-MG cell line but tyrosine-phosphorylated with HGF treatment (Fig. 3).

HGF treatment induces STAT5 activation in the U87-MG cell line. Western blot analysis of nuclear p-STAT5 demonstrated that p-STAT5 was not localized in the nuclei of untreated cells. As shown in Fig. 4, treatment of cells with HGF induced an increase in STAT5 phosphorylation at Tyr-694/699, which reached a maximum within $15 \mathrm{~min}$ and declined toward base-line levels at $4 \mathrm{~h}$ of HGF treatment. Western blot analysis with anti-STAT5 Ab confirmed that similar amounts of STAT5 protein were present following treatment in the absence or in the presence of HGF.

STAT5 SiRNA inhibits the expression of STAT5 and HGF-induced STAT5 phosphorylation in the U87-MG cell line. Fig. 5 presents immunoblots revealing a decrease in STAT5 expression in cells transiently transfected with 

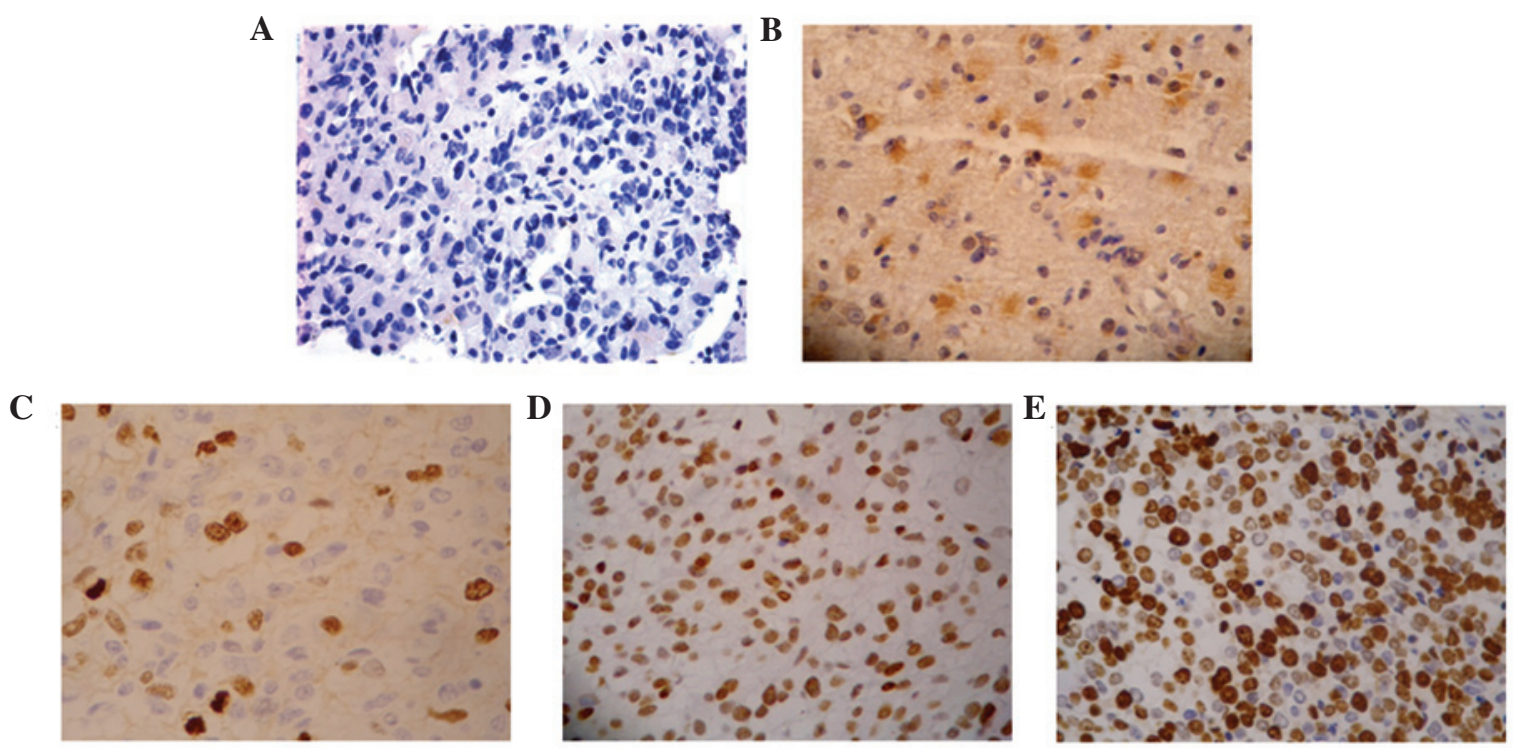

Figure 2. Immunohistochemical staining of p-STAT5 in glioma samples. (A) Negative control; (B) non-neoplastic brain tissue; (C) grade II glioma; (D) grade III glioma; (E) grade IV glioma. p-STAT5, phosphorylated signal transducers and activators of transcription 5.

A

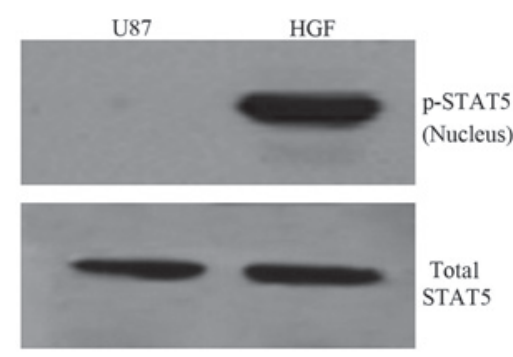

B

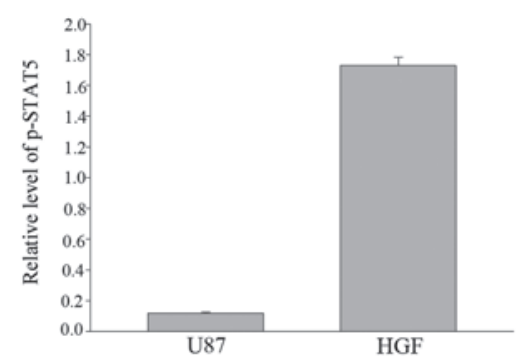

Figure 3. HGF induces activation of STAT5 in U87-MG cells. (A) Western blot analyses of nuclear p-STAT5 protein in HGF-stimulated and resting U87-MG cells. (B) Normalized densitometric analysis of p-STAT5 protein levels. Data are expressed as the mean \pm standard error, $n=3$. STAT5, signal transducers and activators of transcription 5; HGF, hepatocyte growth factor.

STAT5 siRNA, whereas STAT5 expression was not altered by control siRNA. As another control, the ability of STAT5 siRNA to inhibit STAT5 activation induced by HGF was also assessed. It was demonstrated that STAT5 siRNA effectively inhibited HGF-induced STAT5 phosphorylation. These results suggested that siRNA may be a useful tool in limiting STAT5 expression and activation.

HGF and STAT5 siRNA induce changes in expression of downstream cell-cycle regulators at the transcriptional level. To elucidate the effects of HGF and RNAi treatment on STAT5 downstream genes, the expression of CyclinD1, $\mathrm{p} 21^{\mathrm{Cip} 1}$ and $\mathrm{p} 27^{\mathrm{Kipl}}$ were examined by qPCR and western blot
A

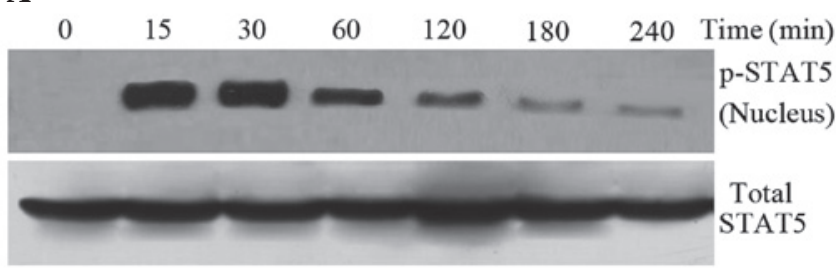

$\mathbf{B}$

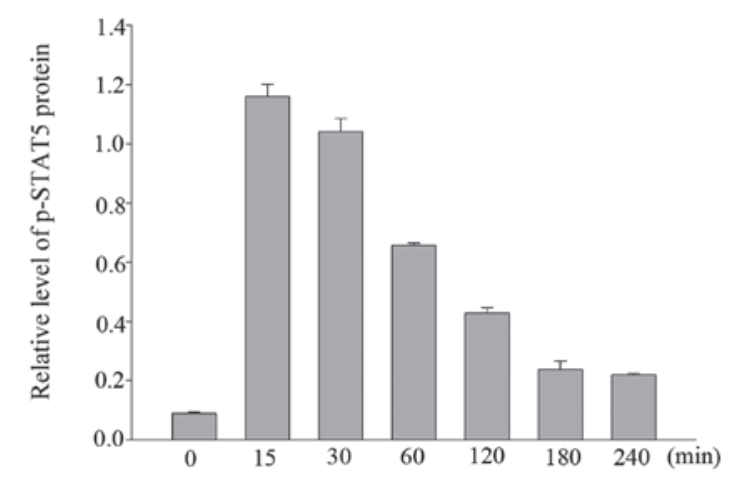

Figure 4. STAT5 is activated by HGF in U87-MG cells after 15 min (A) Western blot analyses of nuclear p-STAT5 protein at different time-points $(0,15,30,60,120,180$ and $240 \mathrm{~min})$ in U87-MG cells following HGF treatment. (B) Normalized densitometric analysis of p-STAT5 protein levels. Data are expressed as the mean \pm standard error, $n=3$. STAT5, signal transducers and activators of transcription 5; HGF, hepatocyte growth factor.

analyses. HGF was able to downregulate the expression of $\mathrm{p} 21^{\mathrm{Cip} 1}$ and $\mathrm{p} 27^{\mathrm{Kipl}}$ and upregulate CyclinD1 at the transcriptional level. To confirm that the effect of HGF on CyclinD1, $\mathrm{p} 21^{\mathrm{Cip} 1}$ and $\mathrm{p} 27^{\mathrm{Kip} 1}$ expression proceeds via STAT5 activation, these cell cycle regulators were assessed in cells that were transiently transfected with STAT5 siRNA. As displayed in Fig. 6, transfection of U87-MG cells with STAT5 siRNA resulted in diminished CyclinD1 and upregulated p $21^{\text {Cipl }}$ and $227^{\text {Kipl }}$ RNA expression even in the presence of HGF, suggesting that this effect is at least in part mediated through STAT5. 
A
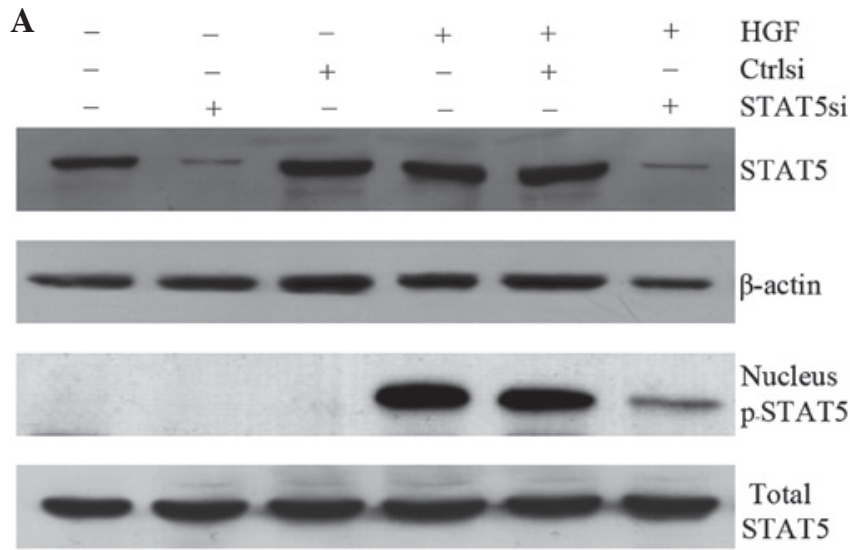

B

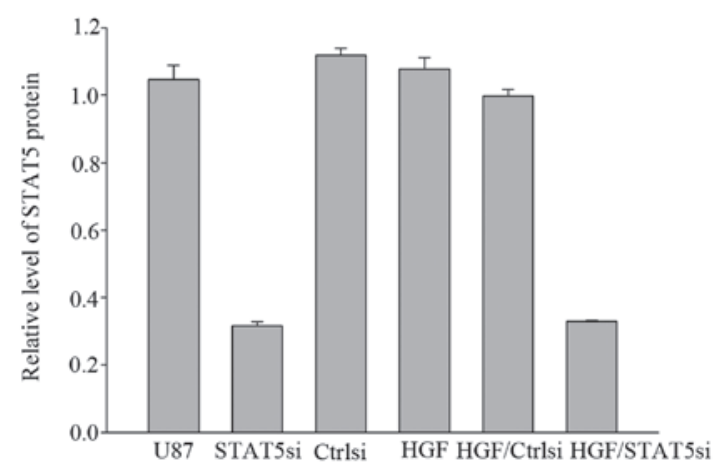

C

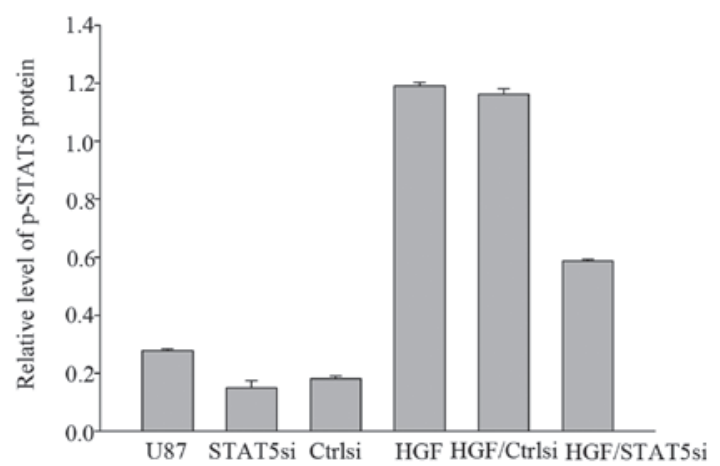

Figure 5. STAT5 siRNA decreases STAT5 protein levels in U87-MG cells and inhibits HGF-induced STAT5 phosphorylation. (A) Western blot analyses of STAT5 and p-STAT5 protein in U87-MG cells treated with HGF and/or STAT5csiRNA. (B) Normalized densitometric analysis of STAT5 and (C) p-STAT5. U87, untransfected; Ctrlsi, transfected with control siRNA; HGF, stimulation with HGF; STAT5si, transfected with STAT5 siRNA. Data are expressed as the mean \pm standard error, $n=3$. STAT5, signal transducers and activators of transcription 5; HGF, hepatocyte growth factor; siRNA, small interfering RNA.

STAT5 is important in the proliferation of U87-MG cells. The results obtained by the MTT assay suggested that HGF is able to act as a growth factor in GBM cells (Fig. 7a). It was demonstrated that HGF caused an increase in the cell number in U-87-MG cells during the entire four-day incubation period. The increase in proliferation for HGF was observed at a concentration of $40 \mathrm{ng} / \mathrm{ml}$, which was demonstrated to stimulate p-STAT5 protein expression. Transfecting U87-MG cells with STAT5 siRNA resulted in a reduction in cell number compared with the control. The viability of U87-MG cells was significantly affected by STAT5 siRNA treatment. Additionally, the viability of U87-MG cells following treatment with $40 \mathrm{ng} / \mathrm{ml} \mathrm{HGF}$ was significantly greater and this increase was suppressed by STAT5 siRNA, but not by control siRNA. The decrease in cell number caused by STAT5 siRNA treatment of GBM cells implies that STAT5 participates in cell cycle progression, cell survival, or both. Cell cycle analysis demonstrated that HGF treatment of U87-MG cells increased the number of cells in $\mathrm{S}$ phase compared with the untreated cells, and that this increase was suppressed by STAT5 siRNA, but not altered by control siRNA. Treating U87-MG cells with STAT5 siRNA resulted in an increased proportion of cells in early G1 phase. This may be due to a G1 phase cell cycle arrest (Fig. 7b).

To evaluate whether CyclinD1 was a genuine target of STAT5, a luciferase reporter assay was performed. As shown in Fig. 8, co-transfection of STAT5 siRNA with the CyclinD1 reporter gene led to significantly decreased CyclinD1 promoter activity, suggesting that STAT5 may target CyclinD1.

\section{Discussion}

Persistent activation of STAT5 occurs in a number of human cancer types, including multiple myelomas, breast, ovarian, prostate carcinomas and head and neck tumors. Human tumors are often characterized by an amplification of either growth factors or cytokines, including interleukin (IL)-2 (6), IL-3 (7), IL-5 (7), IL-7 (8), granulocyte-macrophage colony-stimulating factor (9), insulin (10), erythropoietin (11), thrombopoietin (11), growth hormone (11) and epidermal growth factor (2) that may lead to constitutive activation of STAT5. The activation of STAT5 depends on phosphorylation of a tyrosine residue (Tyr694/699) in the c-terminal domain. Subsequent STAT5 translocation to the nucleus results in transcriptional activation of a variety of genes, including cell cycle regulators CyclinD1, CyclinD2, p2 $1^{\text {Cip1 }}$ and p27 ${ }^{\mathrm{Kip} 1}$ and antiapoptotic genes, including B-cell lymphoma 2 and B-cell lymphoma 2 extra large protein.

A number of studies indicated that STAT5 has a pro-proliferative role in human hepatocellular liver carcinoma, breast cancer, head and neck cancer, prostate cancer and lung adenocarcinoma (12-17). Since STAT5 mediates cancer cell proliferation, identification of factors that increase STAT5 activity is critical to identifying potential therapeutic targets. The mechanisms of STAT5 tyrosine phosphorylation and transcriptional activation may provide important insights into the potential role of STAT5 in the process of tumorigenesis.

The present study demonstrated that STAT5 is constitutively activated in GBM tumors. Furthermore, HGF was able to induce tyrosine phosphorylation of Tyr-694/699 and transcriptional activation of STAT5 in the U87-MG cell line. By using immunofluorescence and western blot analysis, it was also demonstrated that HGF induces nuclear translocation of STAT5 in the U87-MG cell line. This is in agreement with a previous study, which demonstrated that STAT is tyrosine phosphorylated in other cancer cells stimulated by HGF (18). Numerous studies demonstrated that the process of STAT5 phosphorylation is rapid and transient in vitro $(19,20)$. In accordance with these results, the present study demonstrated that HGF promoted the nuclear translocation of STAT5, which was able to be detected within $15 \mathrm{~min}$, reached a 
A
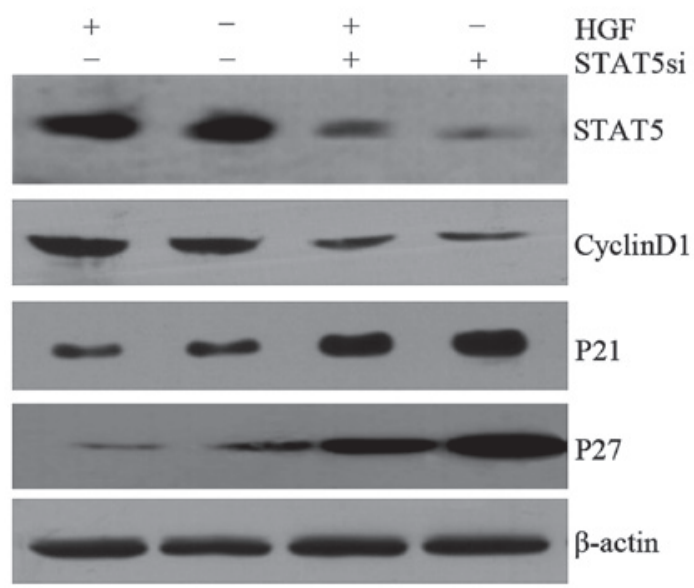

C

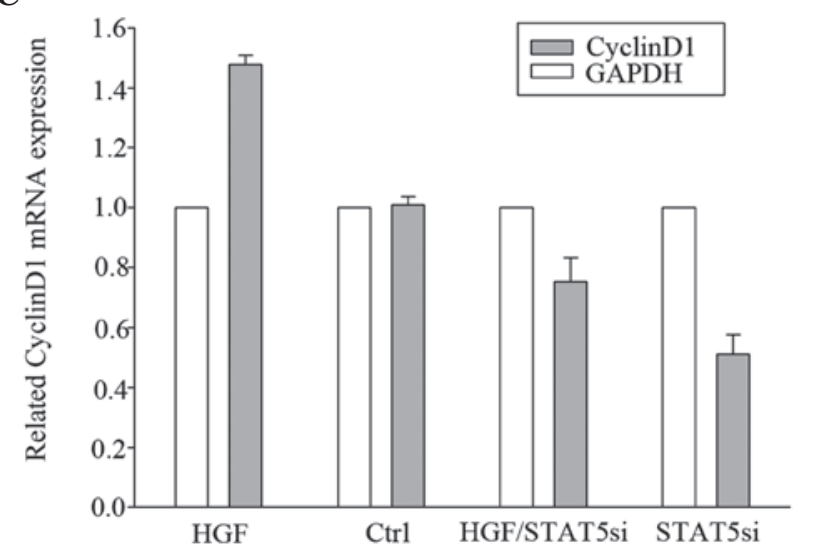

$\mathbf{E}$

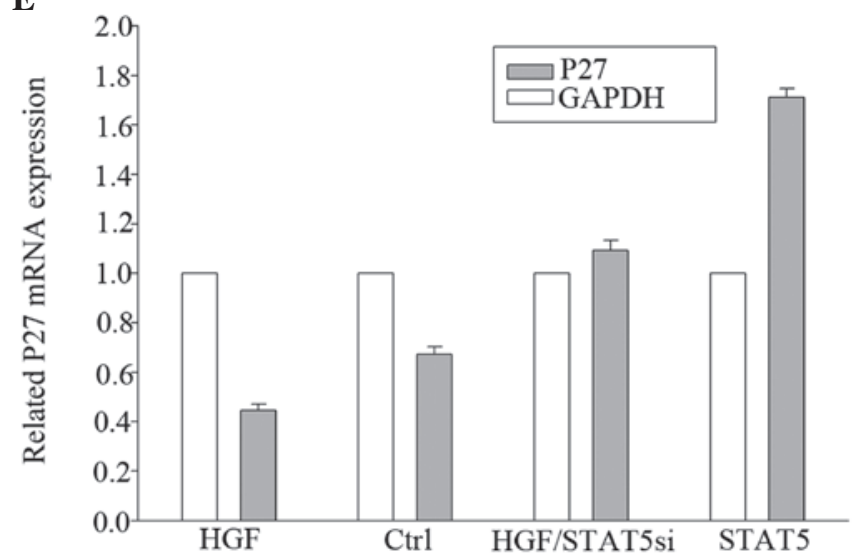

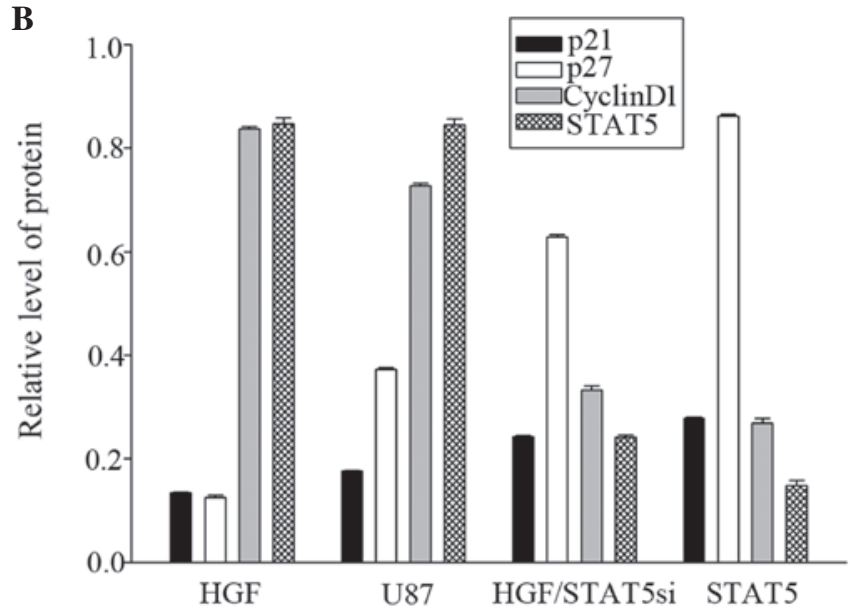

D

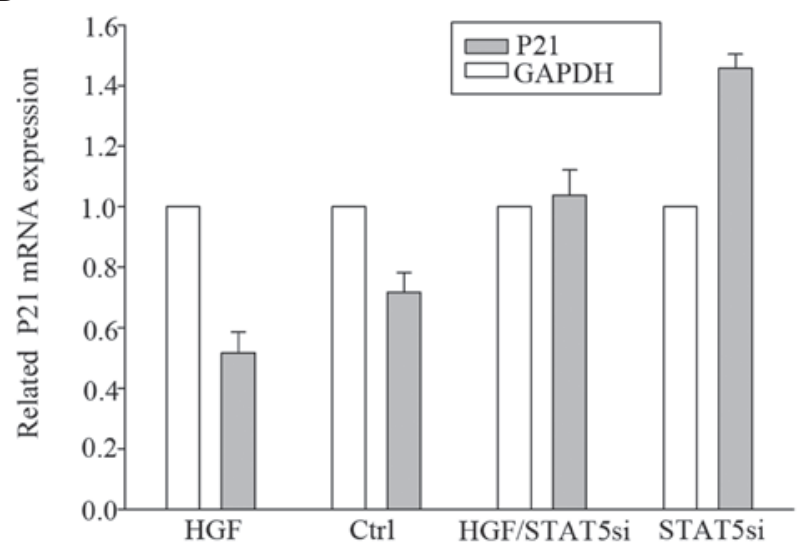

Figure 6. STAT5 siRNA inhibits the expression of CyclinD1 but increases the expression of p21 ${ }^{\text {Cip1 }}$ and p2 ${ }^{\text {Kip1 }}$. (A) Western blot analyses of STAT5, CyclinD1, p21 ${ }^{\text {Cip1 }}$ and $\mathrm{p} 27^{\mathrm{Kip} 1}$ protein in U87-MG cells treated with HGF and/or STAT5 siRNA. (B) Normalized densitometric analysis of STAT5, CyclinD1, p2 ${ }^{\text {Cip1 }}$ and p2 $7^{\text {Kip1 }}$ protein. (C) Normalized quantitative analysis of CyclinD1, (D) p21 ${ }^{\text {Cip1 }}$ and (E) p2 $7^{\text {Kip1 }}$ gene expression using quantitative polymerase chain reaction. Data are expressed as the mean \pm standard error, $n=3$. STAT5, signal transducers and activators of transcription 5; HGF, hepatocyte growth factor; siRNA, small interfering RNA.

maximum and declined toward base-line levels following $4 \mathrm{~h}$ of treatment.

The present study revealed that HGF also exerted proliferative effects in the majority of U87-MG cells. Flow cytometric cell cycle analysis and cell counting revealed that HGF increased the cell number and S-phase fraction. In parallel, increased levels of CyclinD1 were observed, which may explain the increase in cell proliferation. Since CyclinD1 is a downstream target of STAT5, it was expected that increased activity of STAT5 may increase cell growth. Transfection of U87-MG cells with STAT5
siRNA resulted in a diminished effect of HGF on cell proliferation, suggesting that STAT5 is required for HGF-induced cell proliferation in GBM cells.

The characterization of HGF-induced tyrosine phosphorylation and transcriptional activation of STAT5 led to the following conclusions: HGF was able to induce the phosphorylation of STAT5 on Tyr694/699 in the U87-MG cell line and the activated STAT5 (p-STAT5) increased the proliferative ability in the U87-MG cell line, which indicated that STAT5 was necessary for HGF-induced proliferation. These findings suggested 


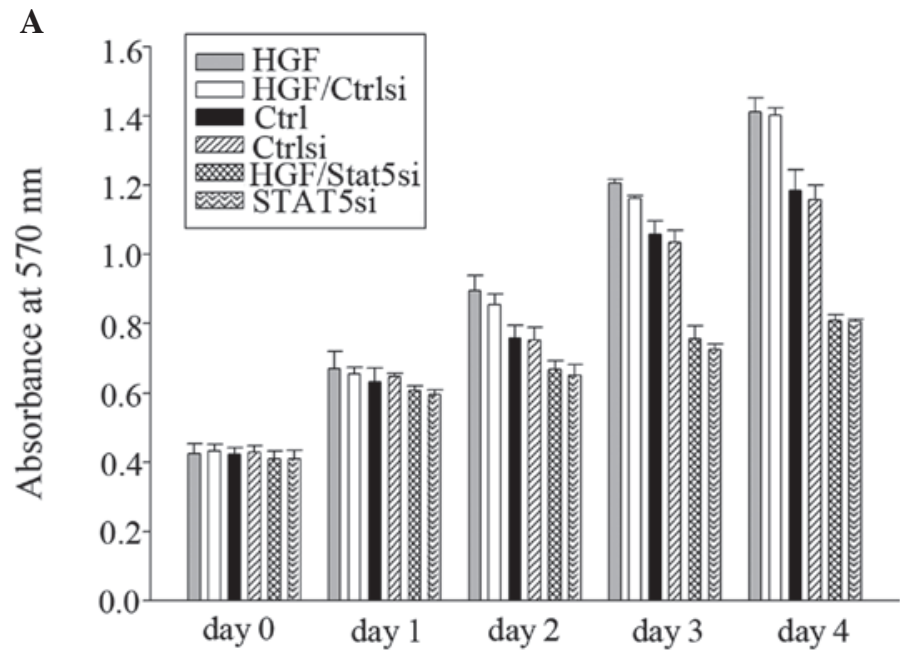

B
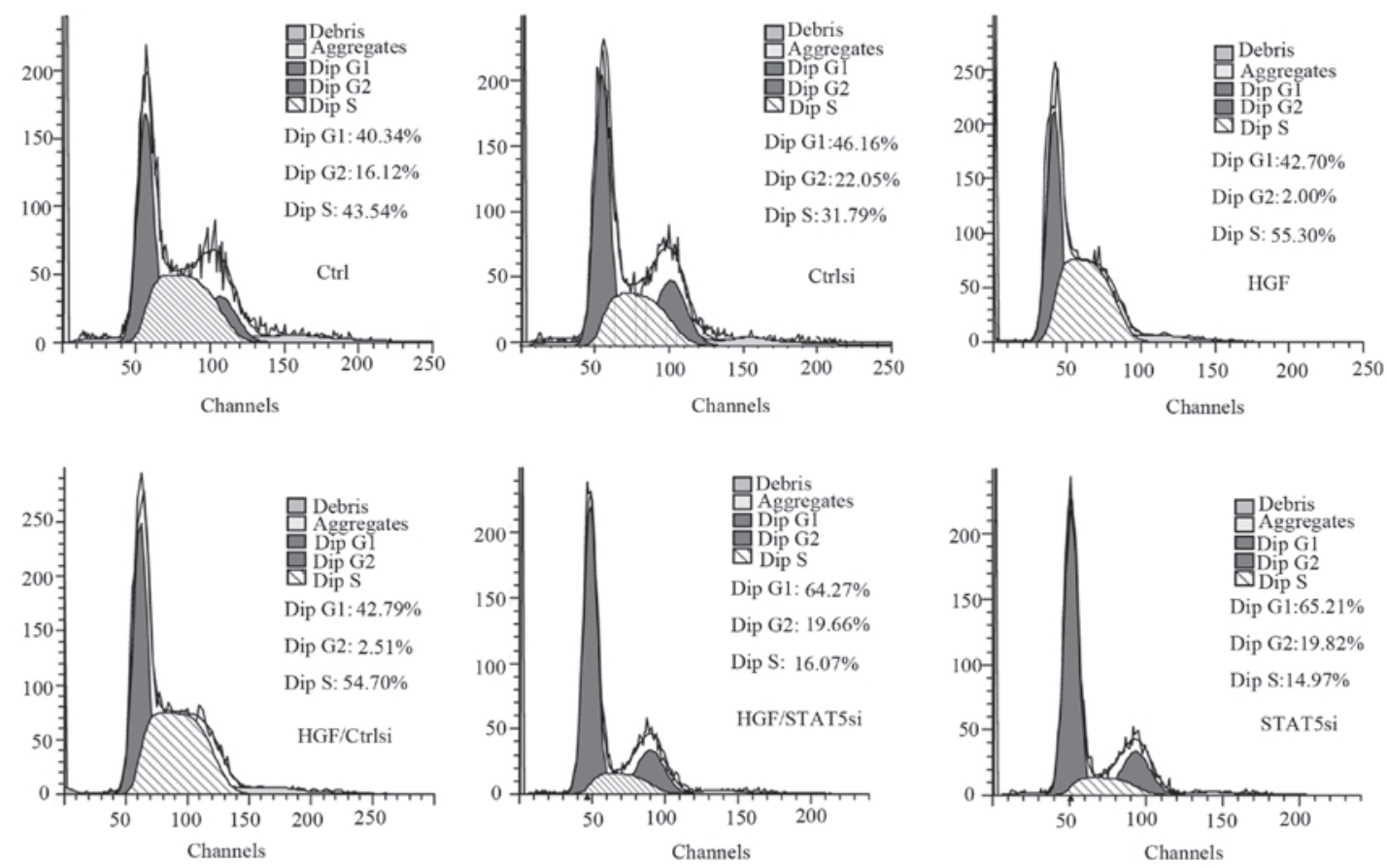

Figure 7. STAT5 siRNA inhibits the proliferation of glioma cells with and without HGF stimulation. (A) MTT assay was used to detect cell proliferation. Data are expressed as the mean \pm standard error, $n=3$. (B) Analysis of cell cycle by flow cytometry. STAT5, signal transducers and activators of transcription 5; HGF, hepatocyte growth factor; siRNA, small interfering RNA.

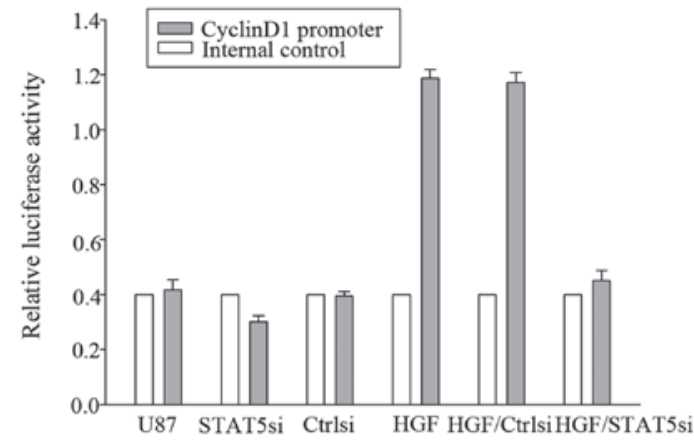

Figure 8. STAT5 siRNA inhibits the transcriptional activity in the CyclinD1 promoter. Luciferase activity assay of pGL3-CyclinD1 vector. Data are expressed as the mean \pm standard error (percentages of relative firefly luciferase activity, normalized to the control group without HGF stimulation, which were normalized to 1 and are representative of six independent groups $(n=3)$. STAT5, signal transducers and activators of transcription 5; HGF, hepatocyte growth factor; siRNA, small interfering RNA. that STAT5 signaling may be a new target for limiting GBM cell proliferation. qPCR analysis determining which transcription units are altered by HGF in a STAT5-dependent manner demonstrated that the expression of CyclinD1 was upregulated; however, $\mathrm{p} 21^{\mathrm{Cip} 1}$ and $\mathrm{p} 27^{\mathrm{Kipl}}$ were diminished upon HGF exposure. These findings suggested a molecular basis for the STAT5 dependence of HGF-mediated GBM progression.

STAT5 pathways were silenced with siRNA in the U87-MG cell line. Suppression of cell growth and a reduced cell number were observed in U87-MG cells following silencing of STAT5. These data support that the STAT5 pathway may serve as a therapeutic target in GBM using siRNA to block the expression of genes encoding STAT5. It has been demonstrated that interference of the STAT5 signaling pathway led to the inhibition of cancer cell proliferation. Silencing of STAT5 in U87-MG cells caused corresponding changes in the cell cycle, which was 
blocked at G1 stage. These data supported that suppression of cell growth in GBM cells is possibly due to the antagonizing effects of silencing STAT5 on cell proliferation that is promoted by elevated STAT5 phosphorylation. Based on these findings, it is hypothesized that GBM cell proliferation is the crucial factor for STAT5 involved in GBM cell tumorigenesis. Certain studies agreed with this hypothesis, which also observed an alteration in STAT5 expression affecting cell proliferation in cancer cells $(21,22)$.

In conclusion, the present study demonstrated for the first time, to the best of our knowledge, that STAT5 was associated with GBM proliferation. STAT5 activation was at least partially mediated by HGF. The present study not only provided a molecular basis for the role of STAT5 in GBM but also suggested a novel therapeutic target for the treatment of GBM.

\section{Acknowledgements}

This study was supported by the Science Foundation of Heilongjiang Health Department (no. 2011-157), the Science Foundation of Heilongjiang Education Department (no. 12521268) and the China Postdoctoral Science Foundation (no. 106941).

\section{References}

1. Buitenhuis M, Coffer PJ and Koenderman L: Signal transducer and activator of transcription 5 (STAT5). Int J Biochem Cell Biol 36: 2120-2124, 2004

2. Cao S, Wang C, Zheng Q, et al: STAT5 regulates glioma cell invasion by pathways dependent and independent of STAT5 DNA binding. Neurosci Lett 487: 228-233, 2011.

3. Zhang H, Li M, Han Y, et al: Down-regulation of miR-27a might reverse multidrug resistance of esophageal squamous cell carcinoma. Dig Dis Sci 55: 2545-2551, 2010.

4. Wang C, Cao S, Yan Y, et al: TLR9 expression in glioma tissues correlated to glioma progression and the prognosis of GBM patients. BMC Cancer 10: 415, 2010.

5. Heim MH: The Jak-STAT pathway: cytokine signalling from the receptor to the nucleus J Recept Signal Transduct Res 19: 75-120, 1999.

6. Hou J, Schindler U, Henzel WJ, Wong SC and McKnight SL: Identification and purification of human Stat proteins activated in response to interleukin-2. Immunity 2: 321-329, 1995.

7. Mui AL, Wakao H, O'Farrell AM, Harada N and Miyajima A: Interleukin-3, granulocyte-macrophage colony stimulating factor and interleukin-5 transduce signals through two STAT5 homologs. EMBO J 14: 1166-1175, 1995.
8. Foxwell BM, Beadling C, Guschin D, Kerr I and Cantrell D: Interleukin-7 can induce the activation of Jak 1, Jak 3 and STAT 5 proteins in murine T cells. Eur J Immunol 25: 30413046, 1995

9. Barahmand-pour F, Meinke A, Eilers A, Gouilleux F, Groner B and Decker T: Colony-stimulating factors and interferon-gamma activate a protein related to MGF-Stat 5 to cause formation of the differentiation-induced factor in myeloid cells. FEBS Lett 360: 29-33, 1995

10. Wartmann M, Cella N, Hofer P, et al: Lactogenic hormone activation of Stat5 and transcription of the beta-casein gene in mammary epithelial cells is independent of p42 ERK2 mitogen-activated protein kinase activity. J Biol Chem 271: 31863-31868, 1996.

11. Pallard C, Gouilleux F, Bénit L, et al: Thrombopoietin activates a STAT5-like factor in hematopoietic cells. EMBO J 14: 2847-2856, 1995.

12. Joung YH, Lee MY, Lim EJ, et al: Hypoxia activates the IGF-1 expression through STAT5b in human HepG2 cells. Biochem Biophys Res Commun 358: 733-738, 2007.

13. Sultan AS, Xie J, LeBaron MJ, Ealley EL, Nevalainen MT and Rui H: Stat5 promotes homotypic adhesion and inhibits invasive characteristics of human breast cancer cells. Oncogene 24: 746-760, 2005

14. Weaver AM and Silva CM: Modulation of signal transducer and activator of transcription $5 \mathrm{~b}$ activity in breast cancer cells by mutation of tyrosines within the transactivation domain. Mol Endocrinol 20: 2392-2405, 2006.

15. Xi S, Zhang Q, Dyer KF, et al: Src kinases mediate STAT growth pathways in squamous cell carcinoma of the head and neck. J Biol Chem 278: 31574-31583, 2003.

16. Xi S, Zhang Q, Gooding WE, Smithgall TE and Grandis JR: Constitutive activation of Stat5b contributes to carcinogenesis in vivo. Cancer Res 63: 6763-6771, 2003.

17. Cao S, Yan Y, Zhang X, et al: EGF stimulates cyclooxygenase-2 expression through the STAT5 signaling pathway in human lung adenocarcinoma A549 cells. Int J Oncol 39: 383-391, 2011.

18. Spiekermann K, Biethahn S, Wilde S, Hiddemann W and Alves F: Constitutive activation of STAT transcription factors in acute myelogenous leukemia. Eur J Haematol 67: 63-71, 2001.

19. Chin H, Nakamura N, Kamiyama R, Miyasaka N, Ihle JN and Miura O: Physical and functional interactions between Stat5 and the tyrosine-phosphorylated receptors for erythropoietin and interleukin-3 Blood 88: 4415-4425, 1996.

20. Pircher TJ, Petersen H, Gustafsson JA and Haldosén LA: Extracellular signal-regulated kinase (ERK) interacts with signal transducer and activator of transcription (STAT) 5a Mol Endocrinol 13: 555-565, 1999.

21. Koppikar P, Lui VW, Man D, et al: Constitutive activation of signal transducer and activator of transcription 5 contributes to tumor growth, epithelial-mesenchymal transition, and resistance to epidermal growth factor receptor targeting. Clin Cancer Res 14: 7682-7690, 2008.

22. Xiong H, Su WY, Liang QC, et al: Inhibition of STAT5 induces G1 cell cycle arrest and reduces tumor cell invasion in human colorectal cancer cells. Lab Invest 89: 717-725, 2009. 